\title{
Visible Light-Mediated [2+2] Cycloaddition for the Synthesis of Azetidines via Energy Transfer
}

\author{
Marc R. Becker, Alistair D. Richardson, and Corinna S. Schindler* \\ Willard Henry Dow Laboratory, Department of Chemistry, University of Michigan, 930 North University Avenue, Ann Ar- \\ bor, Michigan 48109, United States
}

\begin{abstract}
Due to the lack of synthetic methods for their synthesis, azetidines are an underrepresented class of nitrogen-containing heterocycles. Herein, we describe the development of a mild, general protocol for the synthesis of azetidines relying on a visible light-mediated [2+2] cycloaddition between oximes and olefins catalyzed by an iridium photocatalyst. This approach is characterized by its operational simplicity, low catalyst loadings and functional group tolerance. Mechanistic investigations suggest that a triplet energy transfer mechanism is operative.
\end{abstract}

Azetidines ${ }^{1,2}$ are four-membered, nitrogen-containing heterocycles that have seen increased interest in discovery chemistry in recent years. ${ }^{3}$ Cobimetinib $(\mathbf{1})^{4}$, delafloxacin $(\mathbf{2})^{5}$, and baricitinib (3) ${ }^{6}$ are promising lead compounds currently undergoing clinical trials that contain azetidines as core structural motifs (Fig. 1A). Biologically active azetidines, such as penaresidin (4) and mugineic acid (5), have been isolated from natural sources ${ }^{2}$ (Fig. 1A). Compared to their 5- and 6membered ring analogs, azetidines are characterized by their rigid conformations and are known to exhibit superior physicochemical properties as well as metabolic stability. ${ }^{7}$ Despite these desirable characteristics, azetidines are far less prevalent as scaffolds in medicinal chemistry, which is often attributed to a lack of efficient synthetic methods for the construction of these strained, four-membered heterocycles. ${ }^{1}$ Current strategies for the synthesis of functionalized azetidines are most often based on intramolecular substitution reactions with nitrogen nucleophiles, intramolecular alkylations or the reduction of $\beta$-lactams. ${ }^{1,8,9}$ Analogous to the Paternò-Büchi ${ }^{10}$ reaction, in which an excited state carbonyl undergoes a [2+2] cycloaddition with an olefin, an aza Paternò-Büchi reaction between an imine and olefin would provide an efficient way for the synthesis of azetidines. However, in comparison to carbonyls, imines show decreased photoreactivity as a consequence of additional relaxation pathways accessible upon excitation, such as $E / Z$ isomerization. ${ }^{11}$ As a result, successful reports remain scarce and often require rigid imine and olefin-containing systems predisposed for cycloaddition ${ }^{12}$ or cyclic imines bearing electronwithdrawing substituents, such as isoindolones ${ }^{13}$ and azauracils. ${ }^{14,15}$ More recently, the Maruoka group developed an intermolecular [2+2] photocycloaddition between $N$-arylsulfonylimines (6) and styrenes (7) proceeding in up to $67 \%$ yield. (Fig. 1B). ${ }^{16}$ Notably, the reaction was found to be stereospecific, which was attributed to an operative singlet state

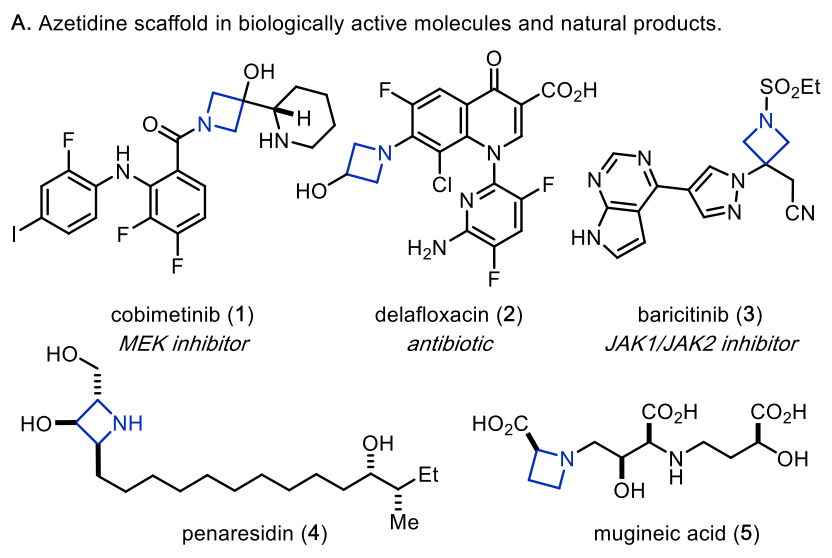

B. Photochemical [2+2] cycloaddition strategies towards functionalized azetidines. Maruoka (2016):

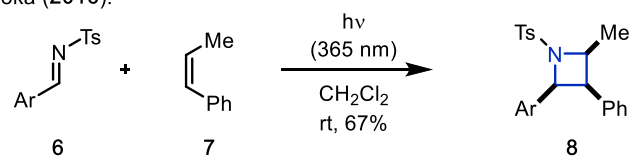

Sivaguru (2017)

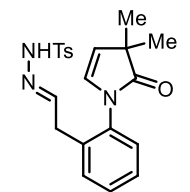

9

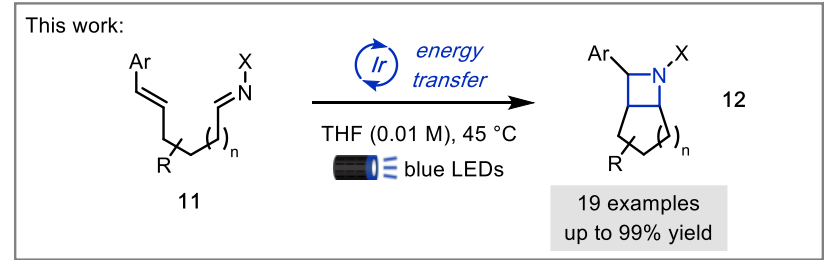

Figure 1. Previous [2+2] cycloaddition strategies towards azetidines and this approach. 
Table 1. Reaction optimization.

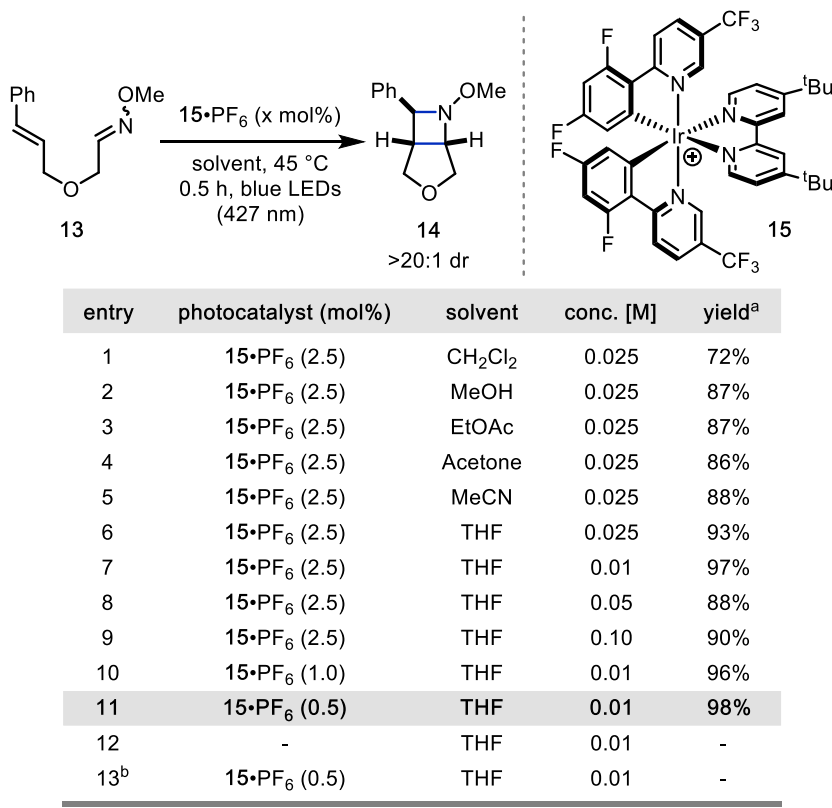

Conditions: Performed on $0.1 \mathrm{mmol}$ scale under irradiation with a blue LED light $(427 \mathrm{~nm})$ for $0.5 \mathrm{~h}$ at ambient temperature; ${ }^{a}$ yield determined by quantitative ${ }^{1} \mathrm{H}$ NMR analysis from the crude mixture using an internal standard; ${ }^{b}$ run in the dark. imine undergoing the photocycloaddition reaction. The Sivaguru group developed an intramolecular aza Paternò-Büchi reaction protocol between oximes or hydrazones and cyclic enamides (9) to form azetidines (10) as cycloaddition products in up to $79 \%$ yield (Fig. 1b). ${ }^{17}$ In contrast to previous photocycloaddition reactions involving imines, the authors propose a triplet sensitization mechanism, in which a triplet state enamide initiates the photocycloaddition after energy transfer from a sensitizer. However, the relatively high triplet energy of enamides limits the reaction to triplet sensitizers such as xanthone $\left(E_{\mathrm{T}}=74 \mathrm{kcal} \mathrm{mol}^{-1}\right)$, which do not absorb in the visible light region and require the use of high-energy UV light. In addition, competing electron-transfer processes can occur that result in catalyst decomposition, reduced yields and limited functional group tolerance. ${ }^{18}$ With the goal to develop a mild and robust [2+2] cycloaddition protocol for the synthesis of azetidines relying on visible light activation, we took inspiration from pioneering work by the Yoon group, who reported the [2+2] cycloaddition of styrenes and dienes to form cyclobutanes via an energy-transfer mechanism. ${ }^{19,20}$ Both styrenes and dienes possess lowest-lying triplet states that are accessible with visible light photocatalysts such as $\operatorname{Ir}\left[\mathrm{dF}\left(\mathrm{CF}_{3}\right) \mathrm{ppy}\right]_{2}(\mathrm{dtbbpy}) \mathrm{PF}_{6}\left(\mathbf{1 5} \cdot \mathrm{PF}_{6}\right) \cdot{ }^{21}$ We hypothesized that a triplet state styrene, accessed through an energy transfer process from a suitable visible light photocatalyst, might be capable of engaging in a [2+2] cycloaddition with a $\mathrm{C}=\mathrm{N}$ double bond to form the azetidine product. Furthermore, the reduced redox potentials of common visible light photocatalysts compared to sensitizers such as xanthone render undesired electron-transfer processes less likely, which would potentially allow for lower catalyst loadings and increased functional group tolerance. With this design principle in mind, we chose oxime 13 (mixture of $E / Z$ oxime isomers) bearing an $(E)$-styrene as a starting point for our investigations. Oximes provide significant advantages over imines as substrates considering their increased stability towards hydrolysis. Irradiation of 13 with a $40 \mathrm{~W}$ blue LED light $(427 \mathrm{~nm})$ in the presence of $2.5 \mathrm{~mol} \% \mathbf{1 5} \cdot \mathrm{PF}_{6}$ resulted in the rapid formation of azetidine $\mathbf{1 4}$ as sole product of the reaction in very good yield and excellent diastereoselectivity $(>20: 1$ d.r. $)$ for a wide range of solvents (Table 1, entry 1-5). By switching to THF as solvent and lowering the concentration, the yield could be further improved (Table 1, entry 7). Remarkably, the catalyst loading could be reduced to loadings as low as $0.5 \mathrm{~mol} \% \mathbf{1 5} \cdot \mathrm{PF}_{6}$ without the need for increased reaction time. Finally, control reactions revealed that both light and photocatalyst were necessary for the $[2+2]$ photocycloaddition to proceed (Table 1, entry 12-13).

With optimized conditions in hand, we next evaluated different oximes and hydrazones for their ability to undergo the [2+2] cycloaddition (Table 2). It should be noted that all substrates were prepared and used as mixtures of $E / Z$ oxime or hydrazone isomers, however, the ratio was found to have no impact on the reaction outcome. $O$-Benzyl oxime $\mathbf{1 6 b}$ converted smoothly to $\mathbf{1 7 b}$ in $96 \%$ yield and very good diastereoselectivity, providing similar results as the $O$-methyl oxime 13. Notably, the reaction can be performed on gram scale at slightly higher concentration with no significant decrease in yield. Free oxime 16c and $N$-Boc hydrazone 16d were reacted under the optimized condition, and the corresponding azetidines $17 \mathbf{c}$ and $\mathbf{1 7 d}$ were isolated in $54 \%$ and $62 \%$ yield, respectively. Interestingly, no reaction was observed with $N, N$-dimethyl hydrazone $\mathbf{1 7 e}$ and only unreacted starting material was recovered from the reaction mixture. Next, we sought to expand the substrate scope of the [2+2] cycloaddition
Table 2. Evaluation of oxime and hydrazone substrates.

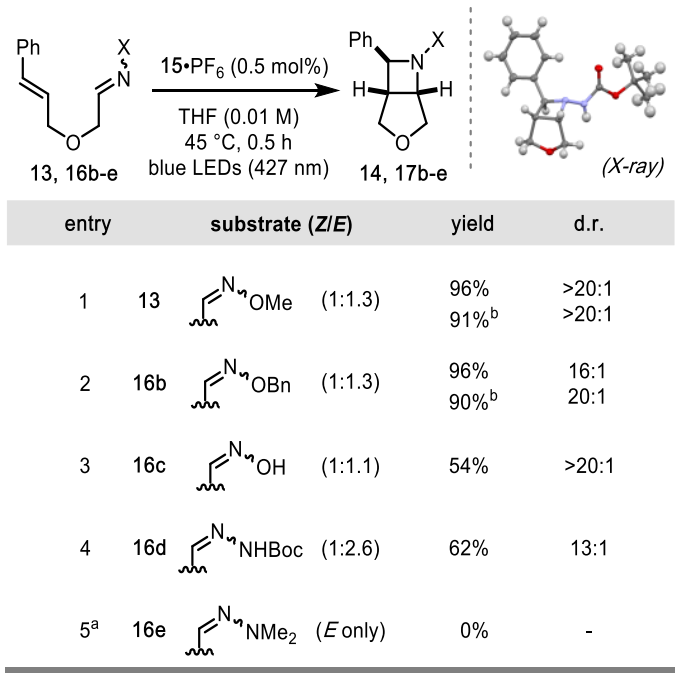

Conditions: Reactions were performed with $0.25 \mathrm{mmol}$ substrate and 0.5 mol\% $15 \cdot \mathrm{PF}_{6}$ in THF $(0.01 \mathrm{M})$ at ambient temperature under blue LED irradiation $(427 \mathrm{~nm})$ for $0.5 \mathrm{~h}$; d.r. was determined by ${ }^{1} \mathrm{H}$ NMR from the crude reaction mixture; ${ }^{a}$ run for $16 \mathrm{~h}$; ${ }^{\mathrm{b}}$ reaction performed on gram scale. 
Figure 2. Scope of the $[2+2]$ cycloaddition reaction.

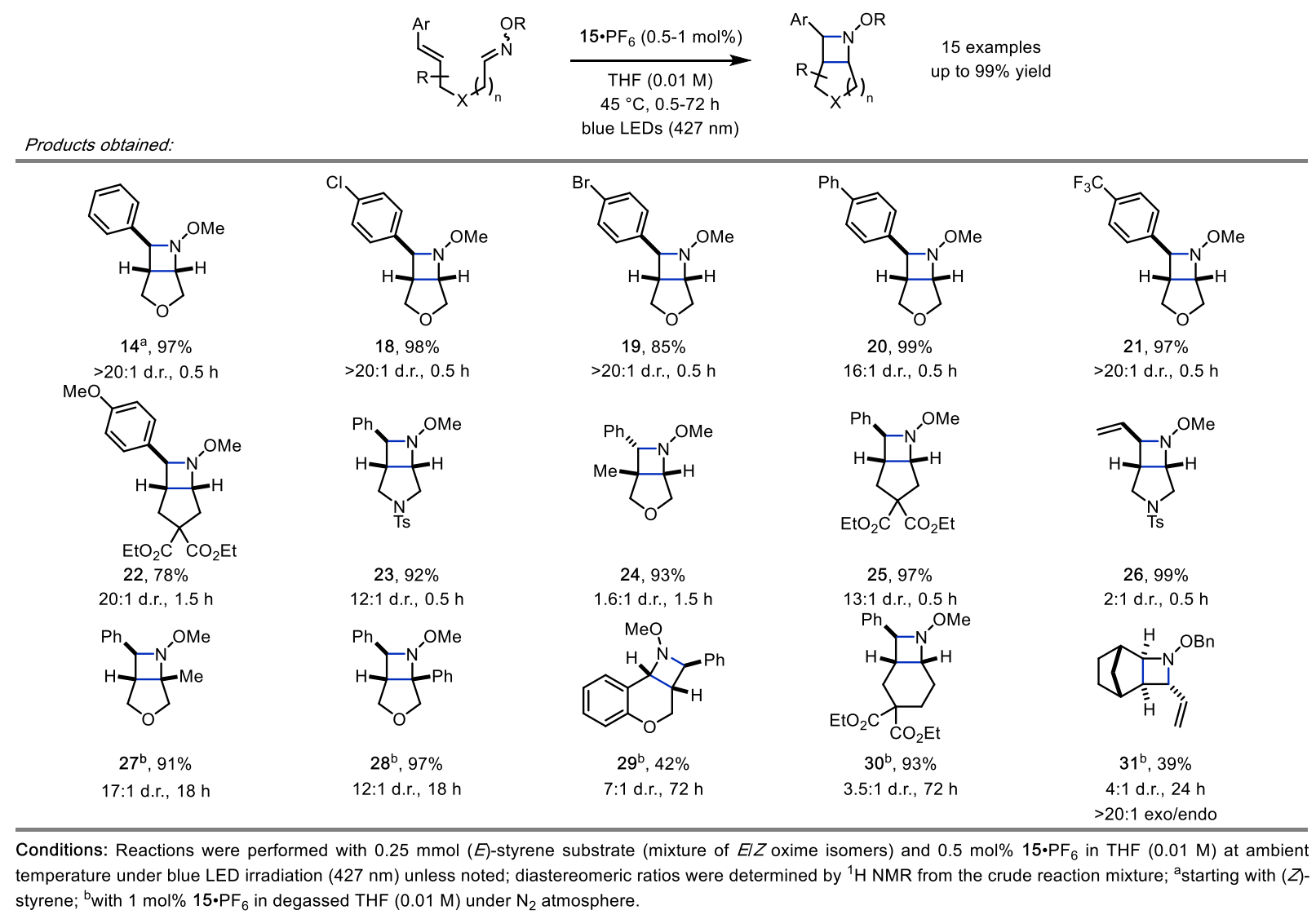

(Fig. 2). In agreement with a mechanism proceeding through a triplet manifold, we observed that $(Z)-\mathbf{1 3}$ gave identical results in the $[2+2]$ cycloaddition as $(E)-\mathbf{1 3}$. This stereoconvergence is characteristic for triplet state styrenes as the same triplet state intermediate is accessed from both the $(E)$ - and $(Z)$-isomer. The optimized conditions proved efficient for a variety of electronically diverse styrenes including both electron-rich and electron-deficient styrenes, affording the corresponding azetidines in excellent yield and diastereoselectivity (18-22). Furthermore, different substitution patterns on the styrene moiety as well as the substrate backbone including esters and sulfonamides were well tolerated (23-25). We also evaluated dienes upon their ability to undergo the developed cycloaddition reaction. Dienes were previously reported by the Yoon group to engage in [2+2] cycloadditions to form cyclobutanes bearing an exocyclic olefin that allows for further functionalization. ${ }^{20}$ After reacting the corresponding diene under the optimized conditions, azetidine $\mathbf{2 6}$ was isolated as the sole product of the reaction in $99 \%$ yield. Notably, we were unable to detect any products arising from a [4+2] cycloaddition, a potential competing side reaction. While the cycloaddition could be readily conducted under ambient atmosphere, we observed significant amounts of solvent decomposition for substrates that required extended reaction times arising from reaction with atmospheric oxygen. However, by simply degassing the reaction mixture prior irradiation and slightly increasing the catalyst loading to $1 \mathrm{~mol} \%$, substrates could be successfully converted to the corresponding products with minimal amounts of solvent decomposition. Under these conditions, oximes derived from methyl and phenyl ketones were converted to the corresponding azetidines $\mathbf{2 7}$ and $\mathbf{2 8}$ in excellent yield. We also evaluated the feasibility of substrates forming azetidines fused to six-membered rings upon [2+2] cycloaddition. These corresponding substrates were found to be significantly less reactive, nevertheless, chromane $\mathbf{2 9}$ and cyclohexane $\mathbf{3 0}$ were obtained after irradiation for 72 hours in $42 \%$ and $93 \%$ yield, respectively. Finally, the developed protocol allows for access of strained bridged bicycles such as $\mathbf{3 1}$ in $39 \%$ yield.

Subsequent efforts focused on investigating the mechanism of the [2+2] cycloaddition reaction. The excited state redox potentials of $15 \cdot \mathrm{PF}_{6}\left(E_{1 / 2}{ }^{\mathrm{III}}{ }^{* / I I}=+1.21 \mathrm{~V} \text { vs. SCE; } E_{1 / 2}{ }^{\mathrm{IV} / I I I^{*}}=-0.89 \mathrm{~V} \text { vs. SCE }\right)^{22}$ are not sufficient for an effective oxidation or reduction of substrate 13, thus, rendering a photoredox process unlikely. Instead, a series of control experiments indicated that a triplet energy transfer mechanism from the photocatalyst to the styrene moiety of the substrate is likely to be operative. Consistent with this hypothesis, when substrate $\mathbf{3 2}$ bearing a terminal olefin with a significantly higher 
triplet state energy than a styrene (approx. $76-84 \mathrm{kcal} \mathrm{mol}^{-1}$ ) was submitted to the standard conditions, no azetidine formation (33) was observed, and unreacted starting material was recovered from the reaction mixture (Fig. 3). ${ }^{23}$ Styrenes possess a triplet energy of approximately $60 \mathrm{kcal} \mathrm{mol}^{-1}$, which suggests that $15 \cdot \mathrm{PF}_{6}\left(E_{\mathrm{T}}=62 \mathrm{kcal} \mathrm{mol}^{-1}\right)$ is capable of sensitizing substrate $\mathbf{1 3}$. On the other hand, the reaction efficiency significantly decreases with photocatalysts that have a triplet energy below $60 \mathrm{kcal} \mathrm{mol}^{-1}$. While $f a c-\operatorname{Ir}(\text { ppy })_{3}\left(E_{\mathrm{T}}=\right.$ $\left.58 \mathrm{kcal} \mathrm{mol}^{-1}\right)$ is still able to mediate product formation, albeit less efficiently, Ru(bpy $)_{3}{ }^{2+}\left(E_{\mathrm{T}}=49 \mathrm{kcal} \mathrm{mol}^{-1}\right)$ does not catalyze the $[2+2]$ cycloaddition at all (Fig. 3). ${ }^{24}$ Next, we conducted a time study to probe for styrene $E / Z$ isomerization under the reaction conditions, a relaxation pathway typically observed for triplet state styrenes. ${ }^{25,26,27}$ Indeed, the time study revealed that $E / Z$ isomerization of pure $(E)-\mathbf{1 3}$ occurs at a similar rate as the productive [2+2] cycloaddition, leading to an initial build-up of $(Z)$-isomer that is eventually converted to the product. This data provides further evidence for the hypothesized triplet sensitization mechanism. Finally, a Stern-Volmer quenching study shows that the styrene moiety of substrate $(E)$-13 is necessary for productive quenching of photocatalyst $15 \cdot \mathrm{PF}_{6}$. In contrast, oxime 34 lacking the styrene functionality but otherwise identical with $\mathbf{1 3}$ does not quench $15 \cdot \mathrm{PF}_{6}$ (Fig. 3). Therefore, a mechanism proceeding through a photosensitized oxime can be ruled out, which confirms our initial reaction design as the discrete photophysical properties of $\mathbf{1 5} \cdot \mathrm{PF}_{6}$ only allow for selective triplet energy transfer to the styrene, while unproductive processes such as electron transfer to the oxime do not occur.

The developed [2+2] cycloaddition protocol allows for rapid access to highly functionalized azetidines under mild conditions, which can undergo further transformations to result in valuable building blocks (Fig. 4). The oxime $\mathrm{N}-\mathrm{O}$ bond can be readily cleaved with zinc metal under acidic conditions providing 35 in $87 \%$ yield. Furthermore, hydrogenolysis conditions facilitate reductive cleavage of the azetidine ring to generate 3 -aminotetrahydrofuran $\mathbf{3 6}$ in $98 \%$ yield after tosylation of the primary amine.

In summary, we have developed a general and catalytic method for the synthesis of highly functionalized azetidines through a [2+2] cycloaddition between oximes or hydrazones and olefins. The design principle of this transformation relies on an energy transfer mechanism utilizing a visible light photocatalyst, which allows to perform the [2+2] cycloaddition under very mild conditions. We expect that this strategy will provide a new platform for the synthesis of azetidines and will facilitate the development of new enabling [2+2] cycloadditions involving carbon-nitrogen double bonds.

\section{AUTHOR INFORMATION \\ Corresponding author}

*corinnas@umich.edu

\section{ACKNOWLEDGMENT}

We thank the NIH/National Institute of General Medical Sciences (R01-GM118644) for financial support and Dr. Jeff W. Kampf for X-ray crystallographic studies. C.S.S. thanks the Alfred P. Sloan Foundation, the David and Lucile Packard Foundation, and the Camille and Henry Dreyfus Foundation for fellowships.

\section{REFERENCES}

(1) For reviews on azetidines, see: a) Antermite, D.; Degennaro, L.; Luisi, R. Recent advances in the chemistry of metalled azetidines. Org. Biomol. Chem. 2017, 15, 34-50; b) Brandi, A.; Cicchi, S.; Cordero, F.M. Novel Syntheses of Azetidines and Azetidinones. Chem. Rev. 2008, 108, 3988-4035; c) Cromwell, N.H.; Phillips, B. The azetidines. Recent synthetic developments. Chem. Rev. 1979, 79, 331-358. 
(2) Yoda, H.; Takahashi, M.; Sengoku, T. in Heterocycles in Natural Product Synthesis, First Edition, Eds. Majumdar, K.; Chattopadhyay, S.K. Wiley-VCH, 2011.

(3) For recent examples, see: a) Lowe, J.T.; Lee IV, M.D.; Akella, L.B.; Davoine, E.; Donckele, E.J.; Durak, L.; Duvall, J.R.; Gerard, B.; Holson, E.B.; Joliton, A.; Kesavan, S.; Lemercier, B.C.; Liu, H.; Marié, J.-C.; Mulrooney, C.A.; Muncipinto, G.; Welzel-O-Shea, M.; Panko, L.M.; Rowley, A.; Suh, B.-C.; Thomas, M.; Wagner, F.F.; Wei, J.; Foley, M.A.; Marcaurelle, L.A. Synthesis and Profiling of a Diverse Collection of Azetidine-Based Scaffolds for the Development of CNS-Focused Lead-Like Libraries. J. Org. Chem. 2012, 77, 7187-7211; b) Carreira, E.M.; Fessard, T.C. Four-Membered Ring-Containing Spirocycles: Synthetic Strategies and Opportunities. Chem. Rev. 2014, 114, 8257-8322; c) Maetani, M.; Zoller, J.; Melillo, B.; Verho, O.; Kato, N.; Pu, J.; Comer, E.; Schreiber, S.L. Synthesis of a Bicyclic Azetidine with In Vivo Antimalarial Activity Enabled by Stereospecific, Directed $\mathrm{C}\left(\mathrm{sp}^{3}\right)-\mathrm{H}$ Arylation. J. Am. Chem. Soc. 2017, 139, 11300-11306; d) Melillo, B.; Zoller, J.; Hua, B.K.; Verho, O.; Borghs, J.C.; Nelson Jr., S.D.; Maetani, M.; Wawer, M.J.; Clemons, P.A.; Schreiber, S.L. Synergistic Effects of Stereochemistry and Appendages on the Performance Diversity of a Collection of Synthetic Compounds. J. Am. Chem. Soc. 2018, 140, 11784-11790.

(4) Larkin, J.; Ascierto, P.A.; Dréno, B.; Atkinson, V.; Liszkay, G.; Maio, M.; Mandalá, M.; Demidov, L.; Stroyakovskiy, D.; Thomas, L.; de la Cruz-Merino, L.; Dutriaux, C.; Garbe, C.; Sovak, M.A.; Chang, I.; Choong, N.; Hack, S.P.; McArthur, G.A.; Ribas, A. Combined Vemurafenib and Cobimetinib. N. Engl. J. Med. 2014, 371, 1867-1876.

(5) Lemaire S.; Tulkens, P.M.; Van Bebeke, F. Contrasting effects of acidic pH on the extracellular and intracellular activities of the anti-gram-positive fluoroquinolones moxifloxacin and delafloxacin against Staphylococcus aureus. $\mathrm{An}$ timicrob. Agents Chemother. 2011, 55, 649-658.

(6) Fridman, J.S.; Scherle, P.A.; Collins, R.; Burn, T.C.; Li, Y.; Li, J.; Covington, M.B.; Thomas, B.; Collier, P.; Favata, M.F.; Wen, X.; Shi, J.; McGee, R.; Haley, P.J.; Shepard, S.; Rodgers, J.D.; Yeleswaram, S.; Hollis, G.; Newton, R.C.; Metcalf, B.; Friedman, S.M.; Vaddi, K. Selective Inhibition of JAK1 and JAK2 Is Efficacious in Rodent Models of Arthritis: Preclinical Characterization of INCB028050. J. Immunol. 2010, 184, 5298-5307.

(7) St. Jean, D.J.; Fotsch, C. Mitigating Heterocycle Metabolism in Drug Discovery. J. Med. Chem. 2012, 55, 60026020.

(8) For reviews on $\beta$-lactam formation, see: a) Pitts, C.R.; Lectka, T. Chemical Synthesis of $\beta$-Lactams: Asymmetric Catalysis and Other Recent Advances. Chem. Rev. 2014, 114, 7930-7953; b) Alcaide, B.; Almendros, P.; Aragoncillo, C. $\beta$-Lactams: Versatile Building Blocks for the Stereoselective Synthesis of Non- $\beta$-Lactam Products. Chem. Rev. 2007, $107,4437-4492$.

(9) For additional recent strategies towards azetidines, see: a) Reidl, T.W.; Son, J.; Wink, D.J.; Anderson, L.L. Facile Synthesis of Azetidine Nitrones and Diastereoselective Conversion into Densely Substituted Azetidines. Angew. Chem. Int. Ed. 2017, 56, 11579-11583; b) Dowling, M.S.; Fernando, D.P.; Hou, J.; Liu, B.; Smith, A.C. Two Scalable Syntheses of (S)-2-Methylazetidine. J. Org. Chem. 2016, 81, 3031-3036; c) Fritz, S.P.; Moya, J.F.; Unthank, M.G.; McGarrigle, E.M.; Aggarwal, V.K. An Efficient Synthesis of Azetidines with (2-Bromoethyl)sulfonium Triflate. Synthesis 2012, 44, 1584-1590.

(10) For reviews, see: a) D'Auria, M.; Racioppi, R. Oxetane Synthesis through the Paternò-Büchi Reaction. Molecules 2013, 18, 11384-11428; b) D'Auria, M. In CRC Handbook of Organic Photochemistry and Photobiology, $3^{\text {rd }}$ ed.; Eds. Griesbeck, A.G.; Oelgemoller, M.; Ghetti, F.; CRC Boca Raton, 2012, pp 563-681; c) Bach, T. The Paternò-Büchi Reaction of $\mathrm{N}$-Acyl Enamines and Aldehydes - The Development of a New Synthetic Method and its Application to Total Synthesis and Molecular Recognition Studies. Synlett 2000, 1699-1707.

(11) a) Padwa, A. Photochemistry of the carbon-nitrogen double bond. Chem. Rev. 1977, 77, 37-68; b) Pratt, A.C. The photochemistry of imines. Chem. Soc. Rev. 1977, 6, 63-81.

(12) a) Fischer, G.; Fritz, H.; Prinzbach, H. An intramolecular imine/ene - photo-[2+2]-cycloaddition reaction. Tet. Lett. 1986, 27, 1269-1272; b) Marterer, W.; Prinzbach, H. The Azo(Azoxy) Functionality as a $\pi_{2}$ Component in Photo [2+2] cycloadditions 'syn'- and 'anti'-3,4-diazatricyclo[4.2.2.2,5]dodeca-3,7-diene, syntheses, photolyses, X-ray-structure analysis, and PE spectra. Helv. Chim. Acta 1988, 71, 1937-1965; c) Fischer, G.; Fritz, H.; Rihs, G.; Hunkler, D.; Exner, K.; Knothe, L.; Prinzbach, H. Proximate, syn-Periplanar, Rigid Imine(Nitrone)/Ene-, and Diazene(Diazeneoxy)/Ene Systems: Syntheses, Homoconjugate Reactivity and Photochemistry. Eur. J. Org. Chem. 2000, 2000, 743-762.

(13) Howard, K.A.; Koch, T.H. Photochemical reactivity of keto imino ethers. V. $(2+2)$ Photocycloaddition to the carbon-nitrogen double bond of 3-ethoxyisoindolone. J. Am. Chem. Soc. 1975, 97, 7288-7298.

(14) Swenton, J.S.; Hyatt, J.A. Photosensitized cycloadditions to 1,3-dimethyl-6-azauracil and 1,3-dimethyl-6-azathymine. Imine linkage unusually reactive toward photocycloaddition. J. Am. Chem. Soc. 1974, 96, 4879-4885.

(15) For additional examples relying on photochemical [2+2]-cycloadditions, see: a) Nishio, T.; Omote, Y. Photocycloaddition of quinoxalin-2-ones and benzoxazin-2-ones to aryl alkenes. J. Chem. Soc. Perk. Trans. 1 1987, 2611-2615; b) Nishio, $\mathrm{T}$. The $(2+2)$ photocycloaddition of the carbon-nitrogen double bond of quinoxalin-2(1H)-ones to electron- 
deficient olefins. J. Org. Chem. 1984, 49, 827-832; for additional examples relying on catalyzed [2+2] cycloadditions, see: c) Rainoldi, G.; Faltracco, M.; Lo Presti, L.; Silvani, A.; Lesma, G. Highly diastereoselective entry into chiral spirooxindole-based 4-methyleneazetidines via formal [2+2] annulation reaction. Chem. Comm. 2016, 52, 11575-11578; d) Nakamura, I.; Nemoto, I.; Yamamoto, Y.; de Meijere, A. Thermally Induced and Silver-Salt-Catalyzed [2+2] Cycloadditions of Imines to (Alkoxymethylene)cyclopropanes. Angew. Chem. Int. Ed. 2006, 45, 5176-5179; e) Akiyama, T.; Daidouji, K.; Fuchibe, K. Cu(I)-Catalyzed Enantioselective [2+2] Cycloaddition of 1-Methoxyallenylsilane with $\alpha$ Imino Ester: Chiral Synthesis of $\alpha, \beta$-Unsaturated Acylsilanes. Org. Lett. 2003, 5, 3691-3693; f) Uyehara, T.; Yuuki, M.; Masaki, H.; Matsumoto, M.; Ueno, M.; Sato, T. Lewis Acid-Promoted [2+2] Azetidine Annulation of N-Acylaldimines with Allyltriisopropylsilane. Chem. Lett. 1995, 24, 789-790; g) Cabral, J.; Laszlo, P.; Montaufier, M.T. Schizoid reactivity of $N$-benzylidene aniline toward clay-catalyzed cycloadditions. Tet. Lett. 1988, 29, 547-550; h) Aben, R.W.M.; Smit, R.; Scheeren, J.W. High-pressure-promoted [2+2] cycloadditions of imines with electron-rich alkenes. A simple route to azetidines and $\beta$-amino carbonyl compounds. J. Org. Chem. 1987, 52, 365-370.

(16) Sakamoto, R.; Inada, T.; Sakurai, S.; Maruoka, K. [2+2] Photocycloaddition between the Carbon-Nitrogen Double Bonds of Imines and Carbon-Carbon Bonds. Org. Lett. 2016, 18, 6252-6255.

(17) Kumarasamy, E.; Kandappa, S.K.; Raghunathan, R.; Jockusch, S.; Sivaguru, J. Realizing an Aza Paternò-Büchi Reaction. Angew. Chem. Int. Ed. 2017, 56, 7056-7061.

(18) Romero, N.A.; Nicewicz, D.A. Organic Photoredox Catalysis. Chem. Rev. 2016, 116, 10075-10166.

(19) Lu, Y.; Yoon, T.P. Visible Light Photocatalysis of [2+2] Styrene Cycloaddition by Energy Transfer. Angew. Chem. Int. Ed. 2012, 51, 10329-10332.

(20) Hurtley, A.E.; Lu, Z.; Yoon, T.P. [2+2] Cycloaddition of 1,3-Dienes by Visible Light Photocatalysis. Angew. Chem. Int. Ed. 2014, 53, 8991-8994.

(21) For recent examples of styrene and diene photosensitization, see: a) James, M.J.; Schwarz, J.L.; Strieth-Kalthoff, F.; Wibbeling, B.; Glorius, F. Dearomative Cascade Photocatalysis: Divergent Synthesis through Catalyst Selective Energy Transfer. J. Am. Chem. Soc. 2018, 140, 8624-8628; b) Molloy, J.J.; Metternich, J.B.; Daniliuc, C.G.; Watson, A.J.B.; Gilmour, R. Contra-Thermodynamic, Photocatalytic E-Z Isomerization of Styrenyl Boron Species: Vectors to Facilitate Exploration of Two-Dimensional Chemical Space. Angew. Chem. Int. Ed. 2018, 57, 3168-3172; c) Day, J.I.; Singh, K.; Trinh, W.; Weaver, J.D. Visible Light Mediated Generation of trans-Arylcyclohexenes and Their Utilization in the Synthesis of Cyclic Bridged Ethers. J. Am. Chem. Soc. 2018, 140, 9934-9941; d) Zhao, J.; Brosmer, J.L.; Tang, Q.; Yang, Z.; Houk, K.N.; Diaconescu, P.L.; Kwon, O. Intramolecular Crossed [2+2] Photocycloaddition through Visible Light-Induced Energy Transfer. J. Am. Chem. Soc. 2017, 139, 9807-9810.

(22) Prier, C.K.; Rankic, D.A.; MacMillan, D.W.C. Visible Light Photoredox Catalysis with Transition Metal Complexes: Applications in Organic Synthesis. Chem. Rev. 2013, 113, 5322-5363.

(23) Ni, T.; Caldwell, R.A.; Melton, L.A. The relaxed and spectroscopic energies of olefin triplets. J. Am. Chem. Soc. 1989, 111, 457-464.

(24) Strieth-Kalthoff, F.; James, M.J.; Teders, M.; Pitzer, L.; Glorius, F. Energy transfer catalysis mediated by visible light: principles, applications, directions. Chem. Soc. Rev. 2018, 47, 7190-7202.

(25) Singh, K.; Staig, S.J.; Weaver, J.D. Facile Synthesis of Z-Alkenes via Uphill Catalysis. J. Am. Chem. Soc. 2014, 136, 5275-5278.

(26) Metternich, J.B.; Gilmour, R. A Bio-Inspired E-Z Isomerization of Activated Olefins. J. Am. Chem. Soc. 2015, 137, 11254-11257.

(27) Wallentin, C.-J.; Nguyen, J.D.; Finkbeiner, P.; Stephenson, C.R.J. Visible Light-Mediated Atom Transfer Radical Addition via Oxidative and Reductive Quenching of Photocatalysts. J. Am. Chem. Soc. 2012, 134, 8875-8884. 